SAND95-2049 • UC-700

Unlimited Release

Printed August 1995

\title{
CHAPARRAL: A Library for Solving Large Enclosure Radiation Heat Transfer Problems
}

Micheal W. Glass

Prepared by

Sandia National Laboratories

Albuquerque, New Mexico 87185 and Livermore, California 94550

for the United States Department of Energy

under Contract DE-AC04-94AL85000

Approved for public release; distribution is unlimited.

REOEIVED NOV - 69995

OSTI 
Issued by Sandia National Laboratories, operated for the United States Department of Energy by Sandia Corporation.

NOTICE: This report was prepared as an account of work sponsored by an agency of the United States Government. Neither the United States Government nor any agency thereof, nor any of their employees, nor any of their contractors, subcontractors, or their employees, makes any warranty, express or implied, or assumes any legal liability or responsibility for the accuracy, completeness, or usefulness of any information, apparatus, product, or process disclosed, or represents that its use would not infringe privately owned rights. Reference herein to any specific commercial product, process, or service by trade name, trademark, manufacturer, or otherwise, does not necessarily constitute or imply its endorsement, recommendation, or favoring by the United States Government, any agency thereof or any of their contractors or subcontractors. The views and opinions expressed herein do not necessarily state or reflect those of the United States Government, any agency thereof or any of their contractors.

Printed in the United States of America. This report has been reproduced directly from the best available copy.

Available to DOE and DOE contractors from

Office of Scientific and Technical Information

PO Box 62

Oak Ridge, TN 37831

Prices available from (615) 576-8401, FTS 626-8401

Available to the public from

National Technical Information Service

US Department of Commerce

5285 Port Royal Rd

Springfield, VA 22161

NTIS price codes

Printed copy: A03

Microfiche copy: A01 


\title{
CHAPARRAL: A Library for Solving Large Enclosure Radiation Heat Transfer Problems
}

\author{
Micheal W. Glass \\ Manufacturing and Environmental Fluid Dynamics \\ Sandia National Laboratories
}

Albuquerque, NM 87185

\begin{abstract}
Large, three-dimensional enclosure radiation heat transfer problems place a heavy demand on computing resources such as computational cycles, memory requirements, disk $I / O$, and disk space usage. This is primarily due to the computational and memory requirements associated with the view factor calculation and subsequent access of the view factor matrix during solution of the radiosity matrix equation. This is a fundamental problem that constrains Sandia's current modeling capabilities. Reducing the computational and memory requirements for calculating and manipulating view factors would enable an analyst to increase the level of detail at which a body could be modeled and would have a major impact on many programs at Sandia such as weapon and transportation safety programs, component survivability programs, energy programs, and material processing programs. CHAPARRAL is a library package written to address these problems and is specifically tailored towards the efficient solution of extremely large threedimensional enclosure radiation heat transfer problems.
\end{abstract}









\section{Table of Contents}

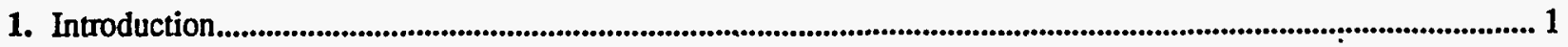

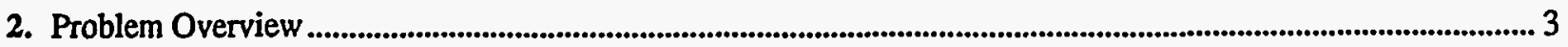

2.1 Enclosure Radiation Heat Transfer and the Radiosity Model .............................................................................3

2.2 Calculation of View Factors ..............................................................................................................................5

2.2.1 The Hemi-Cube Method ............................................................................................................................. 5

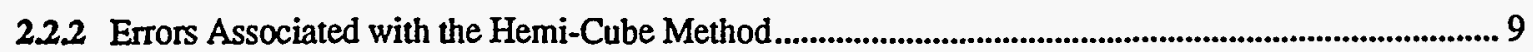

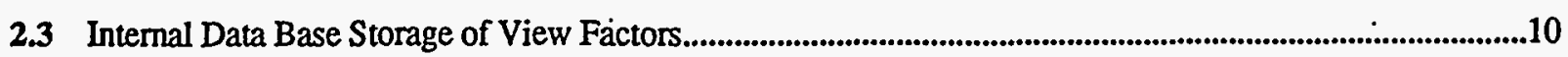

2.4 Solution of the Radiosity Matrix Equation.................................................................................................11

2.4.1 Gauss-Seidel Approach.................................................................................................................. 11

2.4.2 Progressive Refinement Approach ........................................................................................................ 12

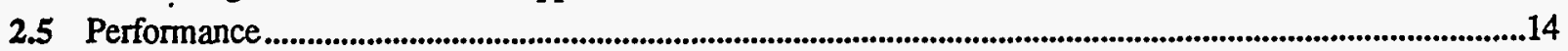

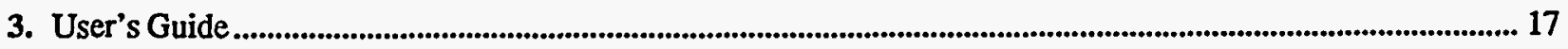

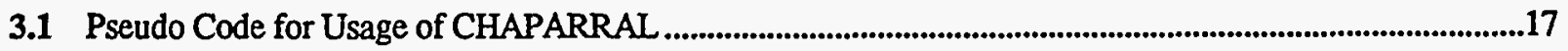

3.2 C Language Interface...........................................................................................................................................18

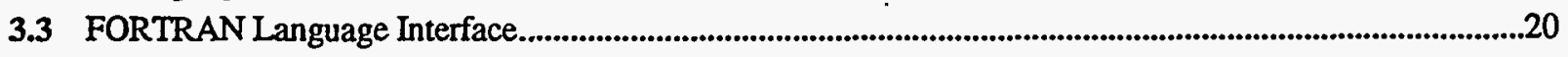

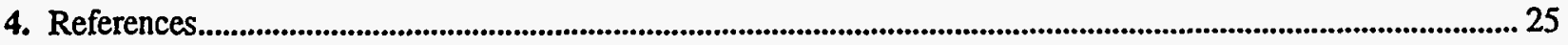

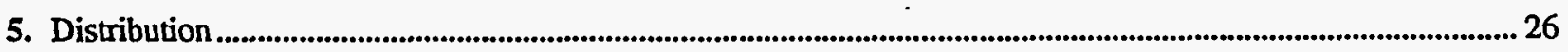




\section{List of Figures}

Figure 1: Geometry and nomenclature for calculating view factors between finite areas. ...........4

Figure 2: Nusselt's hemishere method. ......................................................................................6.

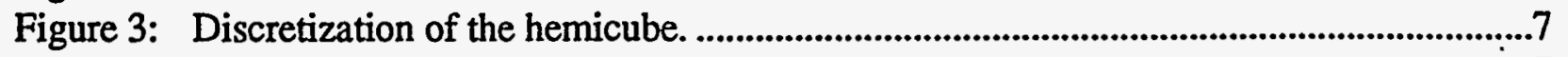

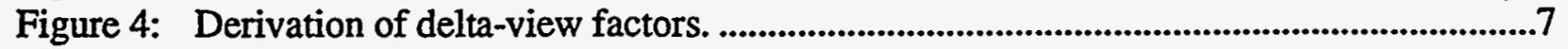

Figure 5: Geometry where the visibility assumption is violated ...................................................9

Figure 6: Example of how aliasing can over or under estimate the projected surface area. ........10

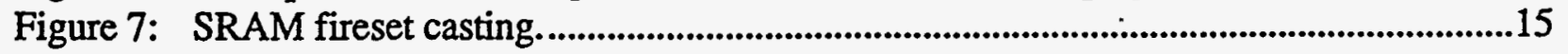




\section{List of Tables}

Table 1: Comparison of FACET with the Hemicube Method ...........................................................14

Table 2: Comparison of Data Compression Schemes................................................................16

Table 3: Comparison of Solution Methods for Solving the Radiosity Matrix Equation ...............16 


\section{Introduction}

Use of the global net radiation method [1] to solve enclosure radiation problems involves the calculation of surface-to-surface view factor pairs. In a standard finite element analysis, the common approach is to use the exposed side of elements as radiating surfaces. To adequately resolve temperature gradients during a finite element thermal analysis, a large number of elements are required in the body being modeled which in turn increases the number of radiating surfaces. Because the number of view factors between the radiating surfaces increases with $O\left(N^{2}\right)$, the calculation of the view factors can easily become a significant, if not dominant, portion of the computation for realistic problems. This is particularly true for steady-state problems or transient problems with a changing geometry or mesh. In many cases, there are problems that we cannot currently solve without sacrificing accuracy (i.e. a less detailed mesh) for reduced computational time.

Depending upon the implementation strategy, efficient solution of large radiation enclosure heat transfer problems typically involves balancing CPU, core memory, and disk I/O requirements for a particular problem. Because there is no set formula for determining how these requirements should be allocated, the experience and intuition of the analyst must be relied upon. CHAPARRAL was written to reduce some of these requirements and allow as much flexibility as possible.

Traditional view factor algorithms from the heat transfer field have proven to be inefficient for very large enclosure radiation problems, particularly when obstructing surfaces are present. However, in the field of computer graphics, new algorithms have been developed for calculating view factors and solving the radiosity problem for a radiosity based global illumination model. One such method for calculating view factors, the hemicube method, has been widely used in the computer graphics field with great success. The hemicube method has been implemented within CHAPARRAL along with more traditional methods for two-dimensional view factor calculations. The hemicube method has demonstrated a tremendous speedup over the traditional double area integration method for three-dimensional geometries. For solution of the radiosity matrix equation, CHAPARRAL includes both a Gauss-Siedel iterative method and a progressive refinement method. For some problems, the progressive refinement method can significantly reduce the number of times the view factors must be accessed, thus speeding up the solution of the matrix equation. Due to the large memory requirements for storage of the view factor matrix, an internal view factor database with compression/decompression algorithms has also been included to reduce the memory requirements and/or disk $\mathrm{I} / \mathrm{O}$ for storing and accessing the view factors. 


\section{Problem Overview}

\subsection{Enclosure Radiation Heat Transfer and the Radiosity Model}

The analysis of thermal radiation heat transfer within an enclosure is complicated because the energy transfer mechanism introduces nonlinearities into the problem and requires the specification of radiation view factors. The energy flux leaving a given surface is composed of directly emitted and reflected energy[1]. The reflected energy flux is dependent upon the incident energy flux from the surroundings, which can be expressed in terms of the energy flux leaving all other surfaces. The energy reflected from surface $k$ is.given by

$$
q_{o k}=\varepsilon_{k} \sigma T_{k}^{4}+\rho_{k} q_{i k}
$$

where $q_{o k}$ is the energy flux leaving the surface, $\varepsilon_{k}$ is the emissivity, $\sigma$ is Boltzmann's constant, $T_{k}$ is the temperature, $\rho_{k}$ is the reflectivity, and $q_{i k}$ is the energy flux incident upon the surface from its surroundings. The amount of incident energy upon a surface from another surface is a direct function of the surface-to-surface view factor, $F_{j k}$. The view factor, $F_{j k}$, can be thought of as the fraction of energy leaving surface $k$ which is incident upon surface $j$. The incident energy flux, $q_{i k}$, can be expressed in terms of the energy flux leaving all other surfaces as

$$
A_{k} q_{i k}=\sum_{j=1}^{N} A_{j} q_{o j} F_{j k}
$$

where $F_{j k}$ is known as the view factor between surface $k$ and surface $j$ (also called form factor, configuration factor). The view factor $F_{j k}$ represents the fraction of energy leaving surface $j$ which is directly incident upon surface $k$. For $N$ surfaces, using the view factor reciprocity relationship gives

$$
A_{j} F_{j k}=A_{k} F_{k j} \quad \text { for } j=1,2,3, \ldots, N
$$

so that

$$
q_{i k}=\sum_{j=1}^{N} F_{k j} q_{o j}
$$

therefore

$$
q_{o k}=\varepsilon_{k} \sigma T_{k}^{4}+\rho_{k} \sum_{j=1}^{N} F_{k j} q_{o j}
$$

Equation (5) can be rewritten as 


$$
B_{k}=E_{k}+\rho_{k} \sum_{j=1}^{N} F_{k j} B_{j}
$$

where $B_{k}$ represents the radiosity of surface $k$ and $E_{k}$ represents the emissivity of surface $k$. This represents $N$ equations which can be recast into matrix form as

$$
\left[\begin{array}{cccccc}
1-\rho_{1} F_{11} & -\rho_{1} F_{12} & \bullet & \bullet & \bullet & -\rho_{1} F_{1 N} \\
-\rho_{2} F_{21} & 1-\rho_{2} F_{22} & \bullet & \bullet & \bullet & -\rho_{2} F_{2 N} \\
\bullet & \bullet & \bullet & \bullet & \bullet & \bullet \\
-\rho_{N} F_{N 1} & -\rho_{N} F_{N 2} & \bullet & \bullet & \bullet & 1-\rho_{N} F_{N N}
\end{array}\right]\left[\begin{array}{c}
B_{1} \\
B_{2} \\
\bullet \\
\bullet \\
B_{N}
\end{array}\right]=\left[\begin{array}{c}
E_{1} \\
E_{2} \\
\bullet \\
\bullet \\
E_{N}
\end{array}\right]
$$

or

$$
\mathbf{K B}=\mathbf{E}
$$

Equation (8) is referred to as the radiosity model or radiosity matrix equation. The view factor between two finite surfaces $i$ and $j$ is given by

$$
F_{i j}=\frac{1}{A_{i}} \int_{A_{i} A_{j}} \frac{\cos \theta_{i} \cos \theta_{j}}{\pi r^{2}} \delta_{i j} d A_{j} d A_{1}
$$

where $\delta_{i j}$ is determined by the visibility of $d A_{j}$ to $d A_{i} . \delta_{i j}$ is equal to one if $d A_{j}$ is visible to $d A_{i}$ and is equal to zero otherwise. See Figure 1 for the geometric interpretation of the individual terms.

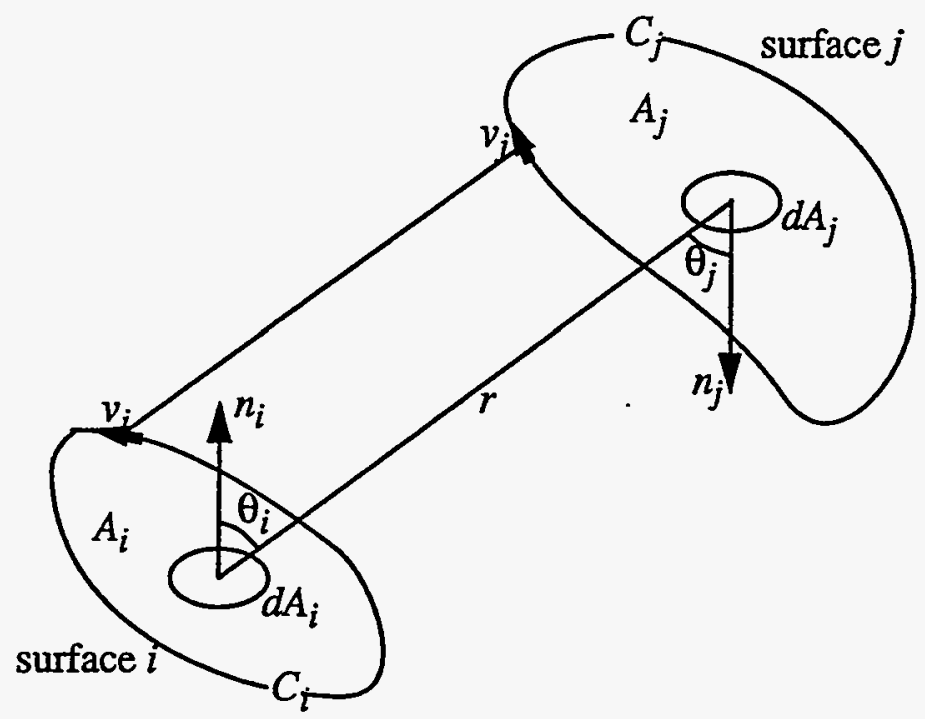

Figure 1 Geometry and nomenclature for calculating view factors between finite areas. 
As mentioned earlier, enclosure radiation heat transfer problems usually require computer resources of the following nature: (1) CPU processing to calculate the view factors and solve the radiosity matrix equation; (2) core memory for storage of the view factors; and (3) disk storage and I/O for out-of-core storage of the view factors and accessing the view factors when solving the radiosity matrix equation. Efficiently solving problems of this nature requires balancing these three needs to achieve optimum performance. CHAPARRAL addresses these three areas and provide some improvement in performance. CHAPARRAL consists of three main sections: (1) calculation of the geometric view factors; (2) an internal database for storing and accessing the view factor matrix; and (3) solution of the radiosity matrix equation. In this chapter, the implementation of each of these areas will be discussed in greater detail.

\subsection{Calculation of View Factors}

Emery et. al. [2] provide an excellent review of the various methods commonly used to calculate view factors. Each has their advantages and disadvantages as detailed in the paper. Therefore, for greater flexibility, multiple algorithms are available within CHAPARRAL for view factor computations. Traditional methods were made available by modifying the FACET [3] package to be a subroutine library and incorporating it into CHAPARRAL. FACET uses traditional methods such as line integration and double area integration and handles 2D planar, 2D axisymmetric, and 3D geometries. An implementation of the hemicube algorithm is available for 3D geometries. By default, FACET is used to calculate the view factors for $2 \mathrm{D}$ geometries and the hemicube method is used for 3D geometries. Because the hemicube algorithm is less well known in the heat transfer field as a method to calculate view factors, more detail on this method is provided next.

\subsubsection{The Hemi-Cube Method}

When Goral et. al. [4] first introduced the radiosity method to the computer graphics field, the view factors were calculated using contour line integration; however, even when applied to simple scenes, this was considered a computationally expensive method. For complex geometries with obstructed views, the double area integration scheme had to be used and the time to calculate the view factors became exorbitantly high. With the strive towards real-time image generation, the first significant contribution from the computer graphics field was in the area of view factor calculation. Specifically, the hemicube method for view factor calculation was developed to decrease the computational effort required for such calculations.

The hemicube method is based upon Nusselt's hemisphere analogy and was first proposed by Cohen et. al. in 1985 [5]. Instead of projecting the surfaces onto a hemisphere, a hemicube is used. Nusselt's analogy shows that any surface which covers the same area on the hemisphere has the same view factor. From this it is evident that any intermediate surface geometry can be used without changing the value of the view factors (see Figure 2). Hence the hemisphere can be replaced with a hemicube.

The use of a hemicube allows for the development of very efficient algorithms for calculating 


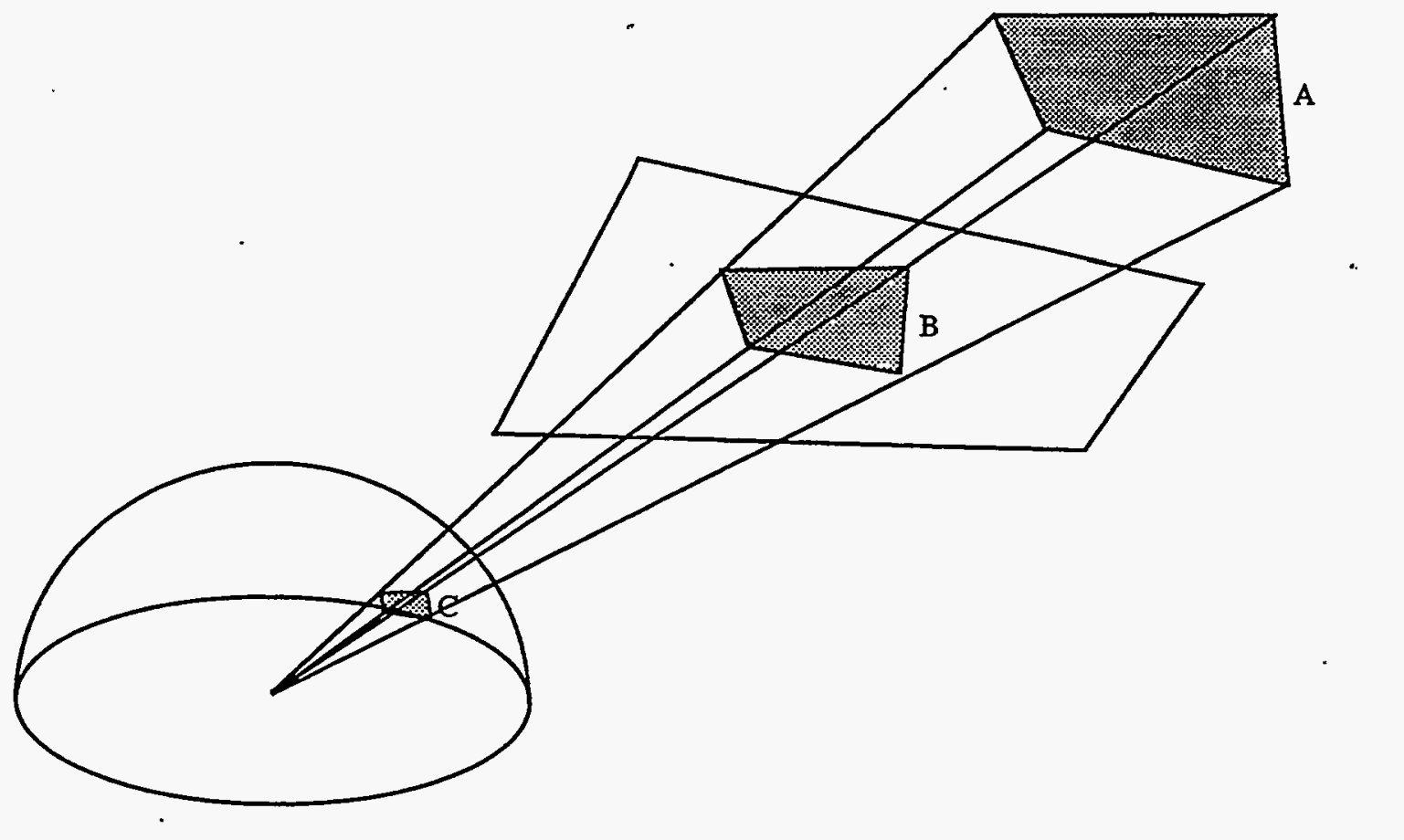

Figure 2 Nusselt's hemishere method.

view factors in a complex environment. The basic algorithm begins by discretizing the surface of the hemicube into a set of $N$ uniform subpatches which will be called pixels. Each pixel defines a particular direction and angle from the receiving patch's centroid. Thus, each pixel contributes a specific delta-view factor value to the overall view factor between two surfaces if the pixel is covered by the projection of the transmitting surface onto the discretized hemicube. See Figure 3 for the geometric interpretation of this method.

The delta-view factors for each pixel, $n=1,2, \ldots, N$ on the hemicube are found from

$$
\Delta \text { viewfactor }=\Delta F_{n}=\frac{\cos \theta_{i} \cos \theta_{j}}{\pi r^{2}} \Delta A_{j}
$$

See Figure 4 for the geometric interpretation of the terms used in Equation (10). The overall view factor is then calculated by

$$
F_{i j}=\sum_{n=1}^{N} \Delta F_{n}
$$

For efficiency, the delta-view factor for each pixel is calculated only once and stored in a lookup table for later use. This lookup table actually only contains values for $1 / 4$ of the top face and 


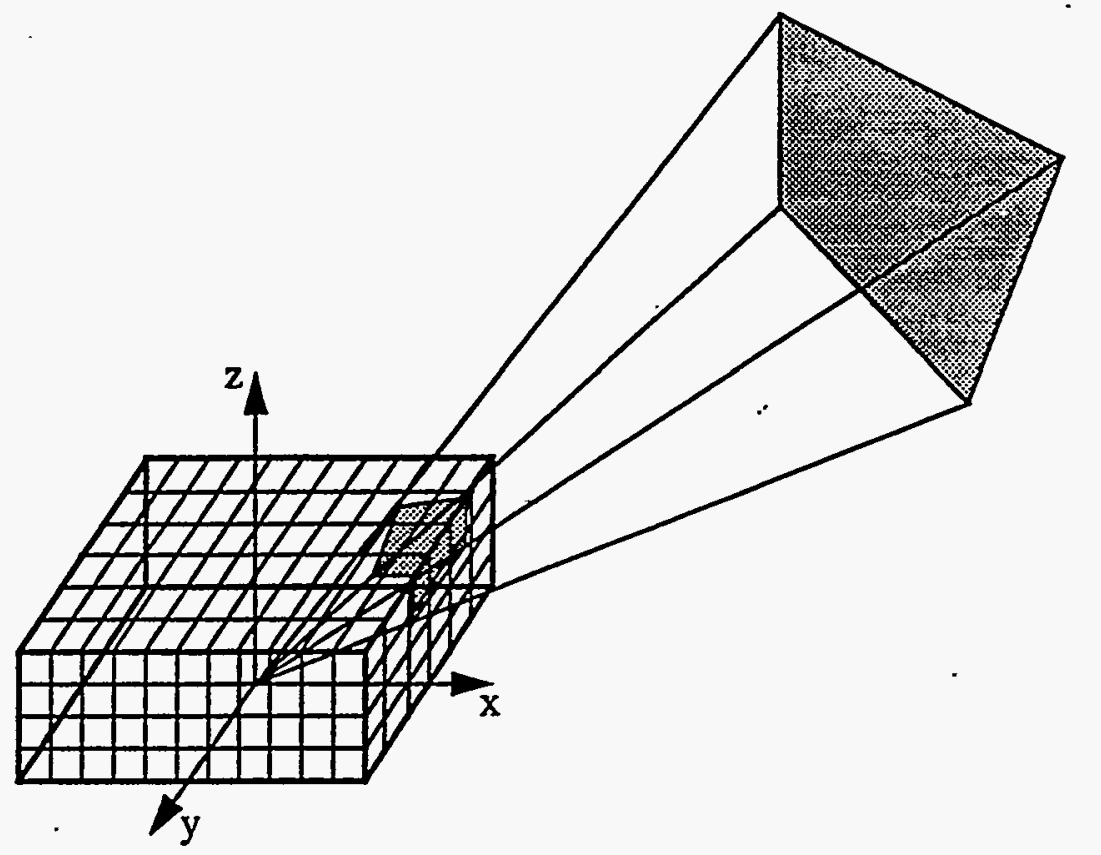

Figure 3 Discretization of the hemicube.

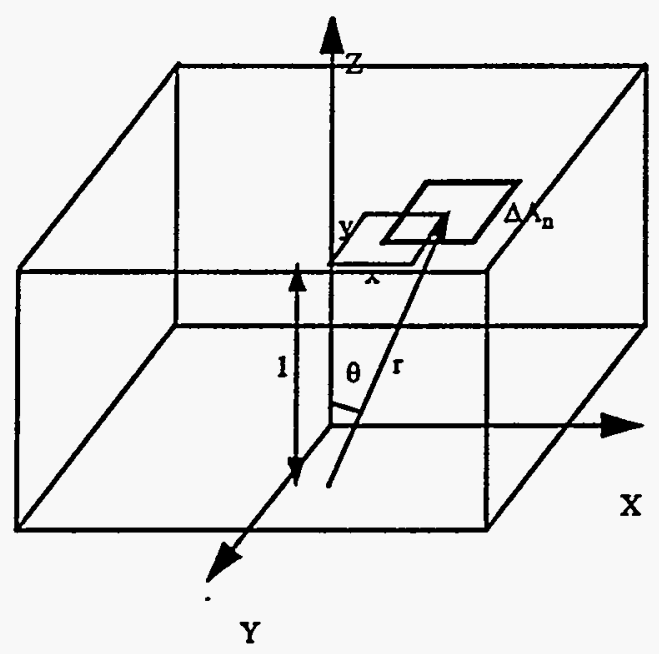

(a)

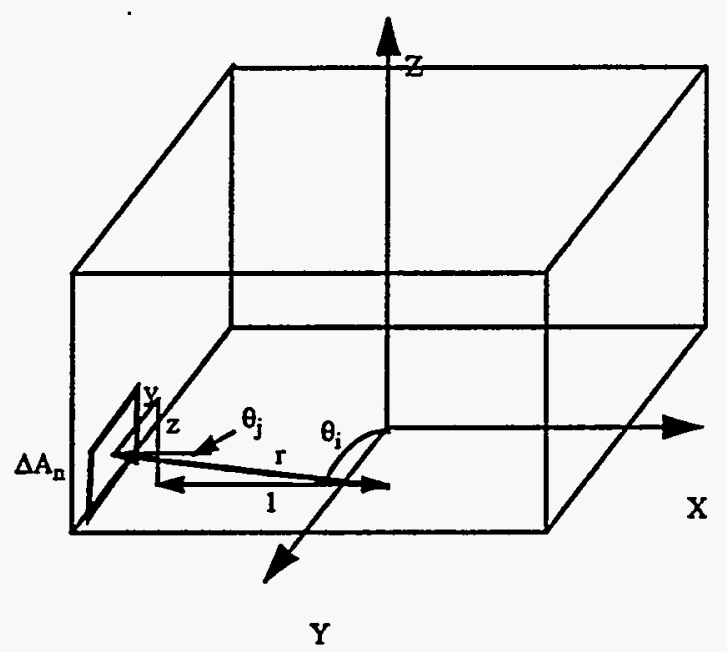

(b)

$$
\begin{gathered}
r^{2}=x^{2}+y^{2}+1 \\
\cos \theta=\cos \theta_{i}=\cos \theta_{j}=\frac{1}{r} \\
\Delta F_{n}=\frac{1}{\pi r^{2}} \Delta A_{n}
\end{gathered}
$$$$
\begin{gathered}
r^{2}=1+y^{2}+z^{2} \\
\cos \theta_{i}=\frac{z}{r} \quad \cos \theta_{j}=\frac{1}{r} \\
\Delta F_{n}=\frac{z}{\pi r^{2}} \Delta A_{n}
\end{gathered}
$$

Figure 4 Derivation of delta-view factors for (a) pixel on top of hemicube and (b) pixel on the side of the hemicube. 
$1 / 2$ of each side face due to symmetry.

The top face of the hemicube represents a $90^{\circ}$ viewing frustum and each side face represents one half of a $90^{\circ}$ viewing frustum which is well known in the field of computer graphics. Hence, all the developments for projecting environments within the frustum can be taken advantage of for computational efficiency.

One can see that instead of calculating view factors on a pair by pair basis, the hemicube algorithm allows for an entire row of view factors $F_{i j}: j=1,2,3, \ldots, N$ to be calculated at the same time after projecting the environment onto the hemicube for surface $i$. Pseudo-code for the hemicube algorithm is given below

Loop on the number of enclosures, $n$

Initialize the hemicube delta view factors

Loop on the number of surfaces, $i$

Calculate surface areas, centroids, and normals

End loop

Loop on the number of surfaces, $i$

Initialize view factor row $F_{i j}=0$ for all $j$

Initialize hemicube ID buffer to NULL surface ID

Initialize hemicube zbuffer to maximum real number

Place hemicube at surface centroid

Loop on number of hemicube sides (including top)

Align view direction with top or side

Loop on number of surfaces, $j$

Project surface jonto the hemicube

Scan convert and zbuffer surface j projection onto hemicube sides

End loop

Sum contribution to $F_{i j}, F_{i j}=F_{i j}+\Sigma \Delta F$ of grid cells with zbuffered ID $=j$

End loop

End loop

End loop 


\subsubsection{Errors Associated with the Hemi-Cube Method}

The hemicube algorithm is based upon various assumptions about the environmental geometry which if violated, will produce inaccurate values for the view factors [6]. The three major assumptions of the hemicube algorithm are: (1) proximity - the distance between surfaces is great. compared to the effective diameter of the surfaces; (2) visibility - the visibility between any two surfaces does not change; and, (3) aliasing - the true projection of each visible surface onto the hemicube can be accurately accounted for by using a finite resolution hemicube.

The proximity assumption is violated whenever surfaces are close compared to their effective diameters or are adjacent to one another. In such cases the distance between the centroid of one surface to all points on the other surface can vary greatly. Because the view factor dependence on distance is nonlinear, the result is a poor estimate of the view factor. The most common violation of this assumption occurs when surfaces are adjoint. Baum, et. al. [6] used a hybrid scheme which combined the hemicube method with an analytical method when the proximity assumption was violated.

The visibility assumption requires that the term $\delta_{i j}$ in Equation (9) remain constant across surface $i$. Because $\delta_{i j}$ is a discontinuous function, the single point evaluation at the centroid of surface $i$ can introduce significant errors to the value of the view factor. This is depicted in Figure 5 where surface 1 has a complete view of surface 2 from its centroid but in fact surface 3 occludes much of surface 2 from surface 1 , In such a case the hemicube algorithm will overestimate $F_{i j}$ by using $F_{d i j}$ calculated from the centroid of surface 1 . To reduce this error, surface 1 could be subdivided into smaller subelements with the hemicube method applied to each subelement and the results combined to produce a more accurate value for the view factor.

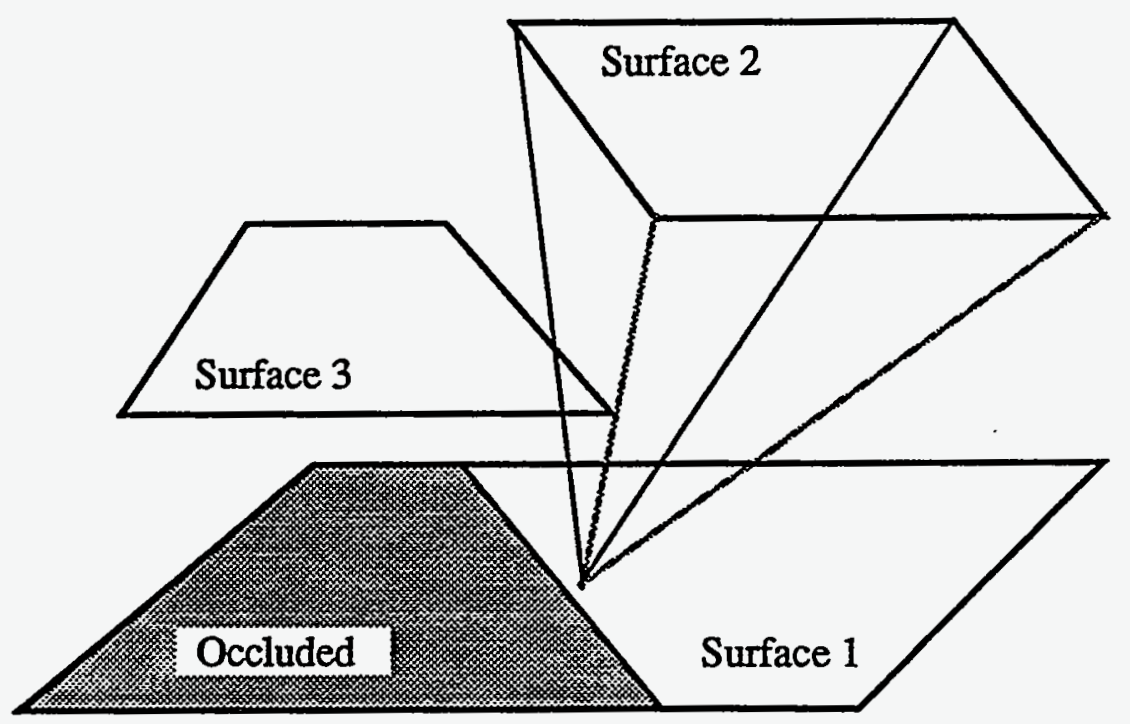

Figure 5 Geometry where the visibility assumption is violated 
The aliasing assumption, that surfaces project exactly onto a whole number of hemicube pixels, is similar to aliasing problems associated with graphical image displays. Because of the finite resolution of the hemicube, the projected areas and resultant view factors may be over or under estimated as shown in Figure 6. Aliasing effects can be reduced by increasing the resolution of the hemicube, by using multiple hemicube subsamples and filtering the results, or by using jittered hemicubes.

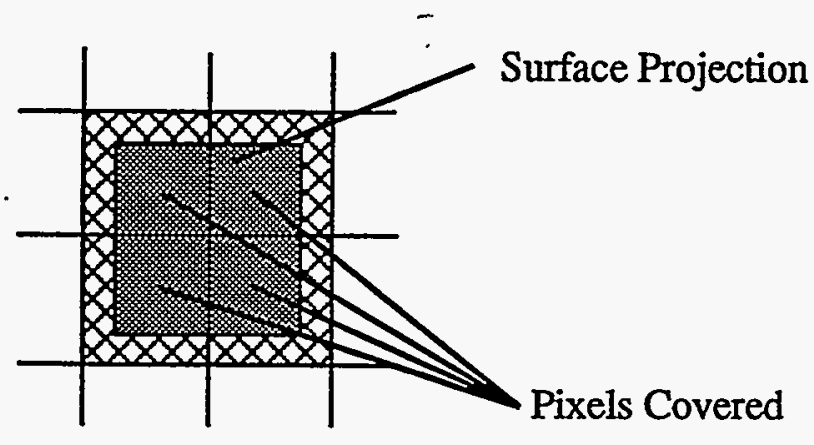

Over Estimate

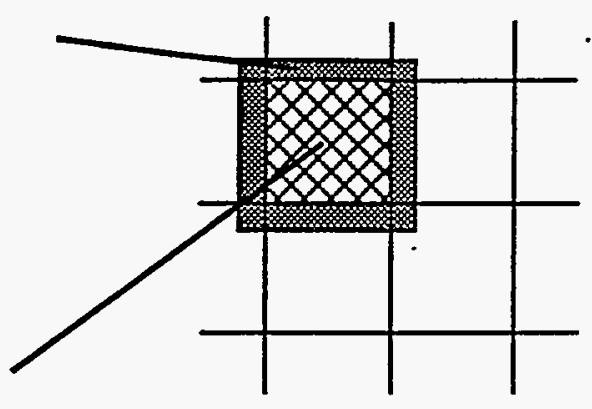

Under Estimate

Figure 6 Example of how aliasing can over or under estimate the projected surface area.

In this initial release of CHAPARRAL, only hemicube jittering has been implemented. With jittered hemicubes, each hemicube is randomly rotated about its parent surface's normal vector. This reduces any preferential alignment of surfaces edges along the hemicube's discretized boundaries.

\subsection{Internal Data Base Storage of View Factors}

When solving radiation heat transfer problems, memory usage can be a major area of concern. The view factor matrix can be either stored in core memory or in a disk file. Depending upon the problem, the view factor matrix may either be sparse or full. If the matrix is stored in full form, one can quickly run into limitations - either core memory limitations or available disk space. For a problem with 5000 participating surfaces, 25 megawords of memory is required to store the entire view factor matrix! From experience, disk I/O (particularly on the Cray or over NFS) is always a bottleneck and should be avoided whenever possible. In CHAPARRAL, disk I/O has been eliminated from the resource balancing equation by allowing the view factors to always be stored in core memory. Though storing the view factors in core memory eliminates the disk $\mathrm{I} / \mathrm{O}$ bottleneck, one can quickly run into core memory limitations. On Sandia's Cray/YMP, processes are limited to 32 megawords of core memory usage so this limit can be easily exceeded on problems of more than 5000 participating surfaces. On other systems without this memory limit, the memory requirements can still quickly exceed the amount of physical memory which can lead to excessive swapping as virtual memory is accessed. For this reason, when the view factor matrix is stored in memory, CHAPARRAL uses data compression to reduce the required amount of memo- 
ry. The view factor matrix is stored row-by-row and each row is compressed separately. This requires CPU overhead to perform the data decompression each time the view factors are accessed, which adds a new dimension to balancing CPU and memory requirements for a job, but the memory savings can be substantial.

To implement the data compression, an internal database is used to store the view factor matrix and associated parameters. Access to the database is accomplished through a defined Application Programmer's Interface (API). This allows the internal workings of the database to be transparent to the user and supplies a well defined interface for the data compression/decompression routines. The API is described in further detail in Chapter 3.

Several data compression/decompression schemes are available. These include: (1) no compression; (2) byte runlength encoding (BRLE); (3) word runlength encoding (WRLE); and (4) Lempil-Zev-Walsh encoding (LZW). WRLE compression/decompression is available because some computer architectures access words much than faster bytes. Runlength encoding is probably the lowest level compression scheme available. The user's choice of a data compression schemes will depend upon speed/memory requirements of the particular problem.

\subsection{Solution of the Radiosity Matrix Equation}

Solving the radiosity matrix equation requires solving a system of linear equations which, depending upon the geometry of the model, may be dense or sparse. Though there are many methods available for this type of problem, some are better suited than others. Direct methods are not very well suited because of the potentially large size of the problem, thus iterative methods have been used almost exclusively. Of the iterative methods available, Gauss-Seidel has probably been the most widely used; and progressive refinement, one of the newer methods, can be advantageous in certain situations. Both of these methods are available in CHAPARRAL and are briefly described below.

\subsubsection{Gauss-Seidel Approach}

Gauss-Seidel is a variation of the Jacobi method and provides a true relaxation method which usually improves the speed at which the method converges to a solution. This iterative approach converges to a solution by solving the system of equations one row at a time. Physically, this means that the evaluation of the $i t h$ row of equations provides an estimate of the radiosity of surface $i$ based on the current radiosities of all the other surfaces. Hence, the energy leaving surface $i$ is determined by gathering in the energy from the rest of the environment. As such, the solution to the radiosity problem is calculated one surface at a time. Pseudo-code for the algorithm is given below. 
Loop on the number of enclosures

Loop on the number of surfaces

Set $B_{i}=$ starting guess

End loop

While not converged

Loop on number of surfaces, $i$

$B_{i}=E_{i}-\sum_{j=1, j \neq i}^{n} B_{j} \frac{K_{i j}}{K_{i i}}$

End while

End loop

\subsubsection{Progressive Refinement Approach}

As an illumination model, the overwhelming cost of the radiosity method is the computation of the view factors. As with thermal radiation codes, this cost is reduced by calculating the view factors once at the beginning of a calculation and storing them for repeated use in the iteration cycle. In such an environment, the storage cost for the view factors are $O\left(N^{2}\right)$. In 1988, Cohen et. al. [7] proposed a progressive refinement method for the radiosity illumination model which computed the view factors on the fly, thus reducing the storage costs to $O(N)$.

For the progressive refinement approach, the problem is formulated so that the contribution made by surface $i$ to all the other surfaces is calculated. This represents an iterative column solution to the set of radiosity equations. Physically, this means that the energy leaving surface $i$ is shot out into the environment and its effect on all the other surfaces is calculated. Because the hemicube method and basic ray-tracing methods compute the view factors on a row-by-row basis, the reciprocity relationship (Equation 3) must be used to transform a row of view factors into a column of view factors. To converge gracefully and as quickly as possible, the surfaces are presorted according to the energy that each surface has to shoot. Theoretically, the solution is not converged until all the emitted and reflected energy is dispersed throughout the environment. But, the final solution may be approached to within a certain error tolerance very quickly and with only a fraction of the view factors calculated as compared to the full matrix formulation. As an example, Cohen et. al. [7] computed the global illumination of a steel mill scene composed of 30000 surfaces subdivided into 50000 patches. The full matrix formulation would have required the computation of $1.5 \times 10^{9}$ view factors requiring 6 Gbytes of storage space. The progressive refinement method needed only one column of view factors requiring 0.12 Mbytes of storage space. Additionally, the solution converged to an acceptable value after the energy from 2000 surfaces had been shot or less than $5 \%$ of the total number of view factors had been calculated! 
While the progressive refinement approach to solving the radiosity problem has demonstrated tremendous speedups for image synthesis, its usefulness to heat transfer calculations may not have as big an impact. While the method converges rapidly to an acceptable result for image synthesis, are the error tolerances as tight as they would probably be in an engineering calculation or is the converged solution merely a visually acceptable result? Another reason this approach works so well as an illumination model is the fact that in image synthesis there are usually light sources included which have a much larger amount of energy to shoot than the other surfaces. By shooting the energy from the light sources first, and then.from the major surfaces that are impacted with that energy, a very reasonable image can be obtained in a short period of time. But the presence of only a few highly emissive surfaces may not always be the case for heat transfer problems. One has only to look at a metal casting problem as an example where the vast majority of surfaces have emissive powers of the same order. And for transient problems, a breakpoint will exist where it is cheaper to calculate all the view factors first rather than repeatedly calculating a smaller number of view factors for each time step. On the other hand, this approach may prove to be very useful when solving transient problems where the geometry changes with time and hence the view factors will also change and cannot be precomputed for the duration of the calculation. Pseudocode for this method is given below.

Loop on the number of enclosures

Loop on the number of surfaces, $i$

$$
\begin{aligned}
& \text { set } B_{i}=E_{i} \\
& \text { set } \Delta B_{i}=E_{i}
\end{aligned}
$$

End loop

While not converged

Choose surface $i$, such that $\Delta B_{i}{ }^{*} A_{i}$ is largest

Loop on the number of surfaces, $j$

$$
\begin{aligned}
& \text { Set } \Delta \mathrm{rad}=\Delta B_{i}{ }^{*} \rho_{j} F_{j i} \\
& \text { Set } \Delta B_{j}=\Delta B_{j}+\Delta \mathrm{rad} \\
& \text { Set } B_{j}=B_{j}+\Delta \mathrm{rad}
\end{aligned}
$$

End loop

$$
\Delta B_{i}=0
$$

End while

End loop 


\subsection{Performance}

To demonstrate the various aspects of CHAPARRAL, a finite element model of a SRAM fireset casting was used as an example model. This model [8] consists of 3297 elements with 4538 radiating surfaces in 2 enclosures (3495 and 1043 surfaces in the top and bottom enclosures respectively). This model is shown in Figure 7. The three main parts of CHAPARRAL will now be discussed in further detail. All timing values are for a Sun Sparcstation 10/30 workstation.

For flexibility, multiple algorithms are available for view factor computations. The available algorithms include those available in FACET along with an implementation of the hemicube algorithm. By default, FACET is used to calculate the view factors for 2D geometries and the hemicube method is used for 3D geometries. The advantage of using the hemicube method for 3D geometries is illustrated by Table 1 . Comparison of the various data compression schemes is shown in Table 2. From Table 2 it can be seen that for this particular test problem, there is no performance penalty for using WRLE compression. Comparison of the two methods for solving the radiosity matrix equation is shown in Table 3.

Table 1: Comparison of FACET with the Hemicube Method

\begin{tabular}{|c|c|c|c|}
\hline \multirow{2}{*}{ Case Number } & \multirow{2}{*}{$\begin{array}{c}\text { Method Used to } \\
\text { Calculate View } \\
\end{array}$} & Factors & \multicolumn{2}{|c|}{$\begin{array}{c}\text { Time (min) to Calculate } \\
\text { View Factors for: }\end{array}$} \\
\cline { 3 - 4 } & Hemicube & Enclosure 1 & Enclosure 2 \\
\hline 1 & FACET & 15.4 & 1.8 \\
\hline 2 & FACET & 204.1 & 2.8 \\
\hline 3 & FACET & 292.6 & 51.4 \\
\hline 4 & & 12.3 \\
\hline
\end{tabular}

Case 1: $50 \times 50$ hemicube resolution.

Case 2: no blocking surfaces specified, 2 subdivisions per edge.

Case 3: no blocking surfaces specified, auto subdividing on edges.

Case 4: all surfaces specified as potentially blocking, no edge subdivisions. 


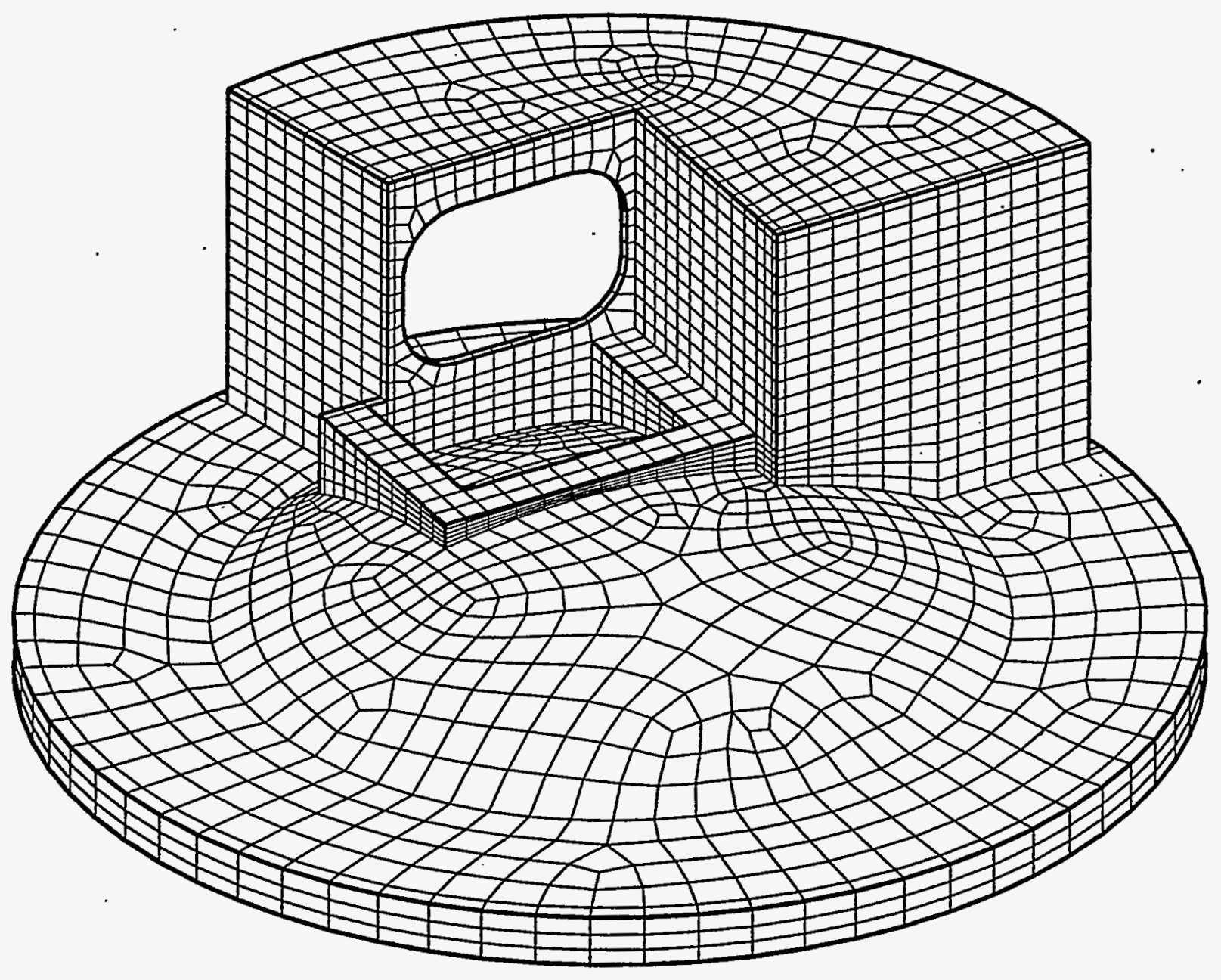

Figure 7 SRAM fireset casting. 
Table 2: Comparison of Data Compression Schemes

\begin{tabular}{|c|c|c|c|c|}
\hline \multirow{2}{*}{ Algorithm } & \multirow{2}{*}{$\begin{array}{c}\text { Total Mb } \\
\text { of Storage }\end{array}$} & \multicolumn{2}{|c|}{$\begin{array}{c}\text { Time (sec) to compress all the } \\
\text { view factor rows for: }\end{array}$} & $\begin{array}{c}\text { Time (sec) to } \\
\text { decompress all the view } \\
\text { factor rows for both } \\
\text { enclosures }\end{array}$ \\
\cline { 3 - 5 } & & Enclosure 1 & Enclosure 2 & \\
\hline None & 51.3 & 5 & 0.5 & 3 \\
\hline WRLE & 13.4 & 5 & 0.7 & 3 \\
\hline BRLE & 12.2 & 15 & 1.8 & 7 \\
\hline LZW & 7.8 & 41 & 5.6 & 25 \\
\hline
\end{tabular}

Table 3: Comparison of Solution Methods for Solving the Radiosity Matrix Equation

\begin{tabular}{|c|c|c|c|}
\hline \multirow{4}{*}{ Solution Method } & \multirow{2}{*}{$\begin{array}{c}\text { Compression } \\
\text { Scheme }\end{array}$} & \multicolumn{2}{|c|}{$\begin{array}{r}\text { Time (sec) to Solve the Radiosity } \\
\text { Matrix Equation for: }\end{array}$} \\
\cline { 3 - 4 } & & Enclosure 1 & Enclosure 2 \\
\hline \multirow{4}{*}{ Gauss-Seidel } & NONE & 107 & 5.3 \\
\cline { 2 - 4 } & WRLE & 100 & 5.7 \\
\cline { 2 - 4 } & BRLE & 150 & 6.0 \\
\cline { 2 - 4 } & LZW & 354 & 32.1 \\
\hline \multirow{4}{*}{$\begin{array}{c}\text { Progressive } \\
\text { Refinement }\end{array}$} & NONE & 69 & 4.8 \\
\cline { 2 - 4 } & WRLE & 70 & 4.9 \\
\cline { 2 - 4 } & BRLE & 80 & 10.2 \\
\cline { 2 - 4 } & LZW & 126 & 11.9 \\
\hline
\end{tabular}




\section{User's Guide}

The CHAPARRAL library is written in both $C$ and FORTRAN with both a $C$ and FORTRAN interface. The routines reside in a non-sharable library called libvf.a. Its location will be dependent upon your installation. On the Department 1500 / Building $880 \mathrm{LAN}$ at Sandia National Labs, it resides in the directory / usr/local/ lib on all the hardware platforms available on the IRN. For internal Sandia use, a standalone program called chaparral is available which accepts COYOTE [9] input files and just calculates the view factors. For users running on multiprocessor, shared memory machines, limited support for multiprocessing is available. Currently, the hemicube method is the only portion of the code that has been written to take advantage of a multi-processing architecture. For the Sun Microsystem's Solaris 2.x operating system, a special version of the library, libvfmt.a exists which utilizes multithreading. The multithreading currently uses Solaris threads. However, when POSIX threads become readily available on other multi-processor architectures, the port should be very easy. When running on a Sun Sparccenter 2000 with $\mathrm{N}$ processors, the standalone chaparral application demonstrates an almost $\mathrm{N}$ times speedup.

Informational output from the CHAPARRAL library, as defined by the debug output level, is written to standard output.

\subsection{Pseudo Code for Usage of CHAPARRAL}

Below is pseudo code outlining the usage of CHAPARRAL. The portions of the pseudo code that are handled by CHAPARRAL are indicated by boldface type.

\section{General code initialization}

Initialize internal view factor database

If view factor matrix has been previously computed

Read view factors into database from pre-existing disk file Else

Loop on the number of enclosures

Calculate view factors for this enclosure

End loop

Write out view factor database to disk file

End if

Loop on number of enclosures

Calculate surface fluxes via Gauss-Siedel or

Calculate surface fluxes via Progressive Refinement 


\section{End loop}

End of code

\subsection{Language Interface}

1. Initialize internal view factor database

init_vf_dbase (num_enclosures, max_surfaces, storage_format)

int num_enclosures total number of enclosures

int max_surfaces maximum \# of surfaces from all the enclosures.

int format compression format: $0=$ no storage, $1=$ no compression, 2 =word run length encoding, 3 =byte run length encoding, and $4=\mathrm{LZW}$ encoding

2. Calculate view factors

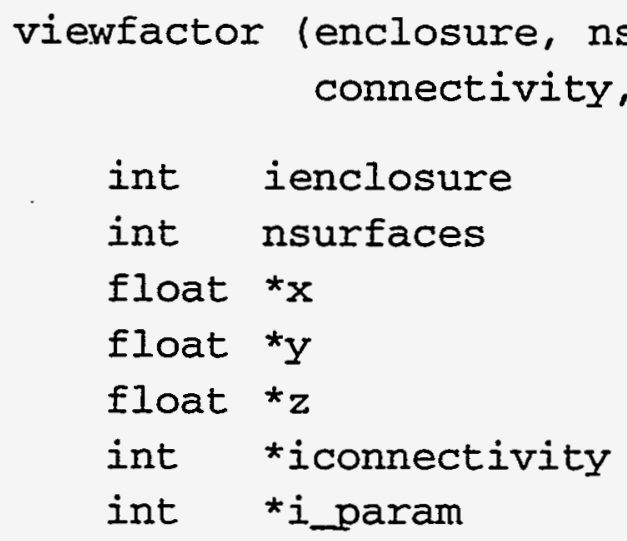

current enclosure number

number of surfaces in this enclosure array of $x$-ccordinates for nodes array of $y$-coordinates for nodes array of $z$-coordinates for nodes surface element connectivity array integer parameter array:

[0] dimensionality of problem: $1=2 \mathrm{D}$ axisymmetric (FACET), 2=2D planar (FACET), 3=3D (hemicube), $3=3 \mathrm{D}$ (FACET)

[1] partial enclosure flag: $0=$ full 


[2] $\begin{aligned} & \text { enclosure, } 1 \text { hemicube resolution, must be an } \\ & \text { even integer }\end{aligned}$
[3] number of nodes i.e. length of $x,$.
[4, and $z$ arrays
[4] least squares smoothing flag:
[5] number of rotations
[6] number of x-grid divisions
[7] number of y-grid divisions
[8] number of z-grid divisions
[9] check for blocking: 0=off, 1=on
[10] maximum number of surface subdi-
[11] debug output level
[12] Number of child processes (or
threads) to use with the hemicube
algorithm
real parameter array:
[0] area of enclosure if this is a
partial enclosure problem

3. Read the view factors from a pre-existing disk file into the internal database read_vfdbase (filename)

$\begin{array}{ll}\text { char *filename } & \text { file name } \\ \text { int format } & \text { storage format: } 0=A S C I I, 1=\text { native ma- } \\ & \text { chine binary, } 2=\mathrm{XDR}, 3=\text { NETCDF (for- } \\ & \text { mats } 2 \text { and } 3 \text { are not yet supported). }\end{array}$

4. Write the view factor matrix to a disk file write_vfdbase (filename, format)

$\begin{array}{ll}\text { char *filename } & \text { file name } \\ \text { int format } & \text { storage format: } 0=\mathrm{ASCII}, 1=\text { native ma- } \\ & \text { chine binary, } 2=\mathrm{XDR}, 3=\mathrm{NETCDF} \text { (for- } \\ & \text { mats } 2 \text { and } 3 \text { are not yet supported). }\end{array}$


5. Solve the radiosity matrix equation

radsolve_gauss (enclosure, radq, tsurf, eps, sigma, . tol, niter, iter)

$\begin{array}{ll}\text { int enclosure } & \text { current enclosure number } \\ \text { float *radq } & \text { array of surface fluxes } \\ \text { float *tsurf } & \text { array of surface temperatures } \\ \text { float *eps } & \text { array of surface emissivities } \\ \text { float sigma } & \text { Stefan-Boltzmann constant } \\ \text { float tol } & \text { convergence tolerance } \\ \text { int niter } & \text { maximum number of iterations } \\ \text { int iter } & \text { if } 0, \text { no convergence reached, else the } \\ & \text { actual number of iterations } \\ \text { radsolve_prog(enclosure, radq, tsurf, eps, sigma, } & \text { iter) } \\ \text { int enclosure niter, } & \text { current enclosure number } \\ \text { float *radq } & \text { array of surface fluxes } \\ \text { float *tsurf } & \text { array of surface temperatures } \\ \text { float *eps } & \text { array of surface emissivities } \\ \text { float sigma } & \text { Stefan-Boltzmann constant } \\ \text { float tol } & \text { convergence tolerance } \\ \text { int niter } & \text { maximum number of iterations } \\ \text { int iter } & \text { if } 0, \text { no convergence reached, else the } \\ & \text { actual number of iterations }\end{array}$

\subsection{FORTRAN Language Interface}

1. Initialize internal view factor database

$\begin{array}{ccc}\text { call INIT_VFDB (num_enclosures, max_surfaces, iformat) } \\ \text { integer } & \text { num_enclosures } & \text { total number of enclosures } \\ \text { integer } & \text { max_surfaces } & \text { maximum number of surfaces } \\ & & \text { from all the enclosures. } \\ \text { integer } & \text { iformat } & \begin{array}{l}\text { compression format: } 0=\text { no } \\ \text { storage, } 1=\text { no compression, }\end{array} \\ & & 2=\text { word run length encoding, } \\ & 3=\text { byte run length encoding, }\end{array}$


2. Calculate view factors

call VUFACTOR(ienclosure, nsurfaces, $x, y, z$, iconnectivity, i_param, r_param)

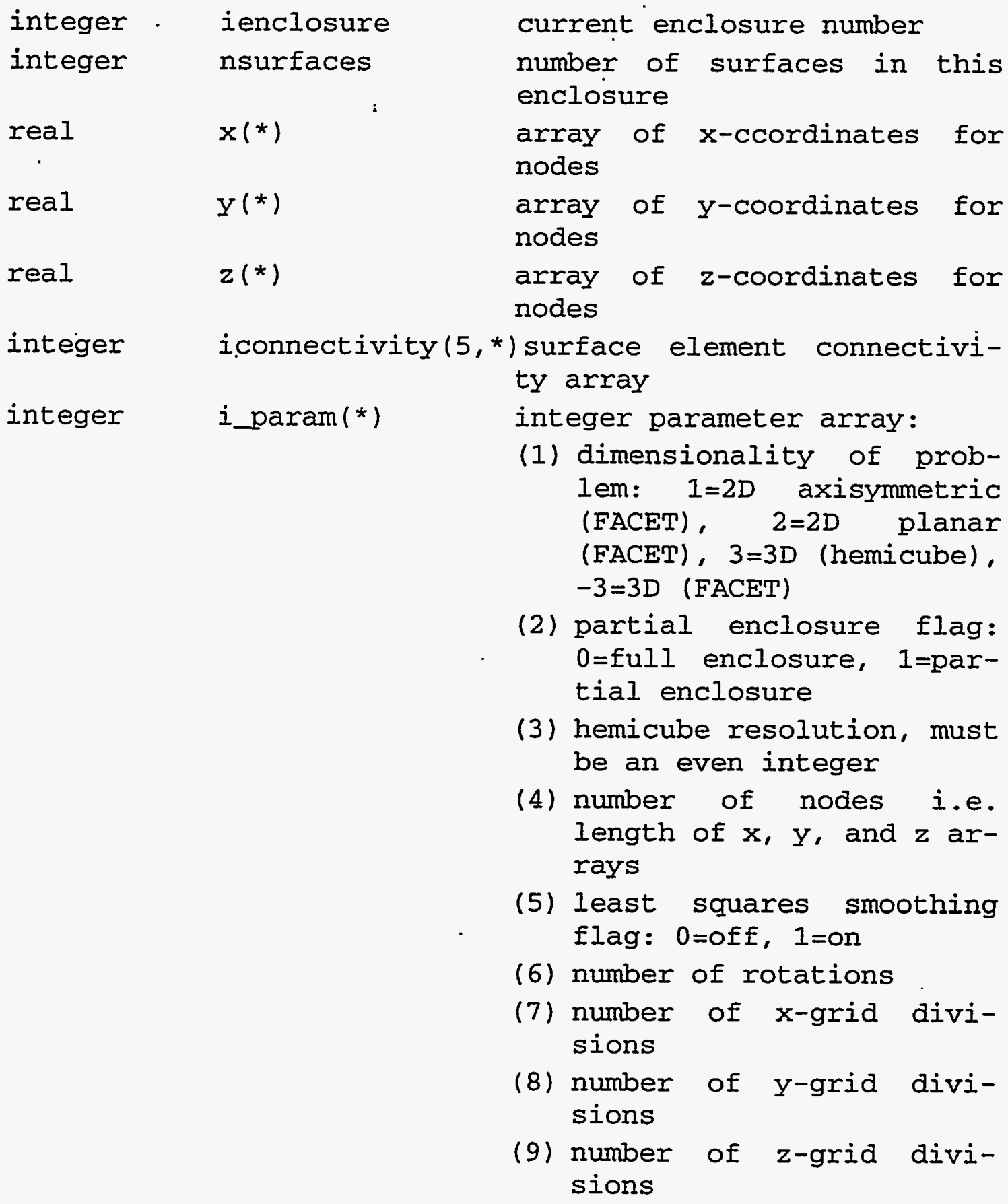




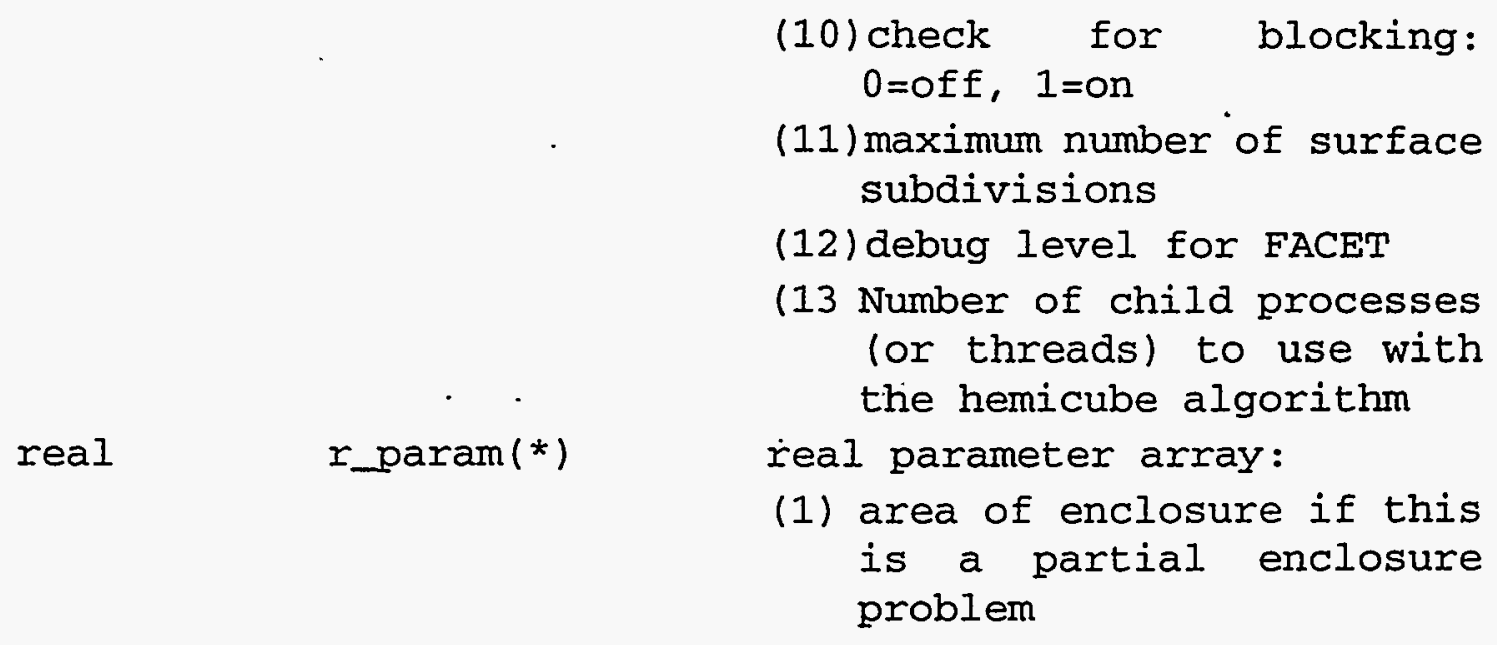

3. Read the view factors from a pre-existing disk file into the internal database

call RD_VFDB (filename)

$$
\text { character* }(*) \text { filename file name }
$$

4. Write the view factor matrix to a disk file

call WR_VFDB(filename, iformat)

$\begin{array}{ll}\text { character* }(*) \text { filename } & \text { file name } \\ \text { integer } & \text { storage format: } 0=A S C I I, \\ & 1=\text { native machine binary, } \\ & 2=X D R, 3=N E T C D F \text { (formats } 2 \\ & \text { and } 3 \text { are not yet supported). }\end{array}$

5. Solve the radiosity matrix equation

call RADSOL_GAUSS(ienclosure, radq, tsurf, eps, sigma, tol, niter, iter)

$\begin{array}{ll}\text { integer } & \text { ienclosure } \\ \text { real } & \operatorname{radq}(*) \\ \text { real } & \text { tsurf(*) } \\ \text { real } & \text { eps(*) } \\ \text { real } & \text { sigma } \\ \text { real } & \text { tol } \\ \text { int } & \text { niter } \\ \text { int } & \text { iter }\end{array}$

current enclosure number array of surface fluxes array of surface temperatures array of surface emissivities Stefan-Boltzmann constant convergence tolerance maximum number of iterations if 0 , no convergence reached, else the actual number of it- 
erations

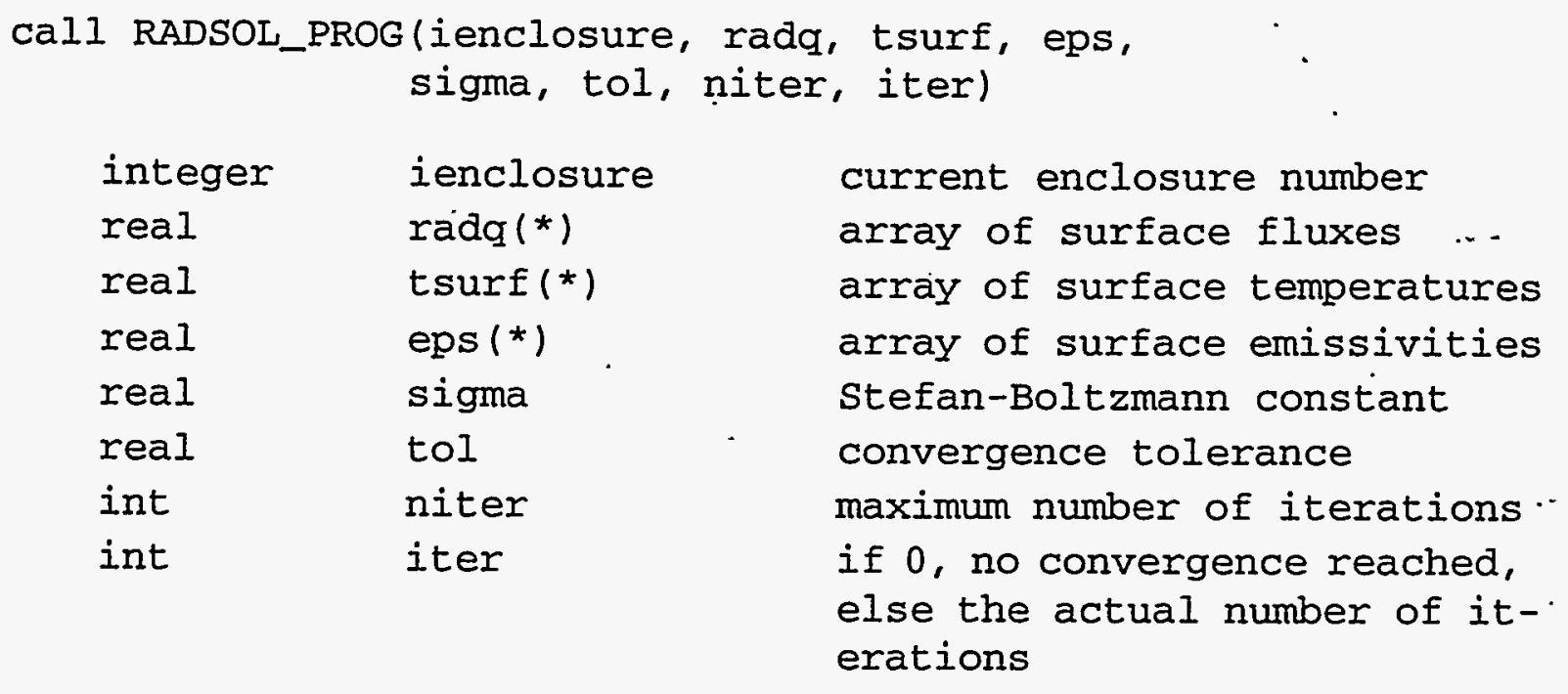




\section{References}

[1] Siegel, R. and Howell, J. R., Thermal Radiation Heat Transfer, McGraw Hill, NY, 2nd Edition, 1981.

[2] Emery, A. F., Johansson, O., Lobo, M., and Abrous, A., "A Comparative Study of Methods for Computing the Diffuse Radiation Viewfactors for Complex Structures," AIAA / ASME / ASCE / ASH 29th SDM Conference, AIAA paper 88-2223, 1988.

[3] Shapiro, A. B., "FACET - A Radiation View Factor Computer Code for Axisymmetric, $2 D$ Planar, and 3D Geometries with Shadowing," Lawrence Livermore Laboratory Technical Report UCID-19887, August 1983.

[4] Goral, C. M., Torrance, K. E., Greenberg, D. P., and Battaile, B., "Modeling the Interaction of Light Between Diffuse Surfaces," Computer Graphics, 18(3):213-222, 1984.

[5] Cohen, M. F. and Greenberg, D. P., "The Hemi-Cube: A Radiosity Solution for Complex Environments, Computer Graphics," 19(3):31-40, 1985.

[6] Baum, D. R., Rushmeier, H. E., and Winget, J. M., "Improving Radiosity Solutions Through the Use of Analytically Determined Form-Factors," Computer Graphics, 23(3):325-334, 1989.

[7] Cohen, M. F., Chen, S. E., Wallace, J. R., and Greenberg, D. P., "A Progressive Refinement Approach to Fast Radiosity Image Generation, Computer Graphics," 22(4):75-84, 1988.

[8] Hogan, R. E., Glass, M. W., Schunk, P. R., and Gartling, D. K., "Thermal Analysis of the Cooling and Solidification of Investment Castings," Proceedings of the $1^{\text {st }}$ International Conf. on Transport Phenomena in Processing, S. I. Guceri, Ed., March 22-26, 1992.

[9] Gartling, D. K. and Hogan, R. E., "Coyote II - A Finite Element Computer Program for Nonlinear Heat Conduction Problems, Part II - User's Manual," SAND94-1179, Sandia National Laboratories, Albuquerque, NM, 1994. 


\section{Distribution}

M. S. Engelman

Fluid Dynamics International 500 Davis Street, Suite 600

Evanston, IL.60201

S. N. Smith

McDonnell Douglas Aerospace 13100 Space Center Blvd Houston, TX 77059-3556
MS 08261514 W. L. Hermina MS 08251515 W. H. Rutledge MS 08331516 MS 04431517 MS 04371518 MS 90438743 MS 9018 8523-2 Central Tech Files MS 089913414 Technical Library (5) MS 061913416 Technical Publications MS 0100 7613-2 Document Processing for DOE/OSTI (10)
C. W. Peterson

H. S. Morgan

R. K. Thomas

M. L. Callabresi
MS 08411500
P. J. Hommert
MS 08331503
J. H. Biffle
MS 08281504
MS 08271511
E. D. Gorham
MS 08271511
MS 08341512 .
MS 08341512
MS 08341512
MS 08341512
MS 08351513
MS 08351513
MS 08351513
D. K. Gartling
M. W. Glass (25)
A. C. Ratzel
M. R. Baer
R. J. Gross
M. L. Hobbs
R. D. Skocypec
D. R. Adkins
R. L. Akau
MS 08351513
B. L. Bainbridge
MS 08351513
E. A. Boucheron
MS 08351513
D. Dobranich
MS 08351513
R. C. Dykhuizen
MS 08351513
S. E. Gianoulakis
MS 08351513
L. A. Gritzo
MS 08351513
D. M. Hensinger
MS 08351513
MS 08351513
R. E. Hogan
R. R. Lober
MS 08351513
MS 08351513
V. F. Nicolette
V. J. Romero
M. P. Sherman
MS 08351513
S. R. Tieszen 



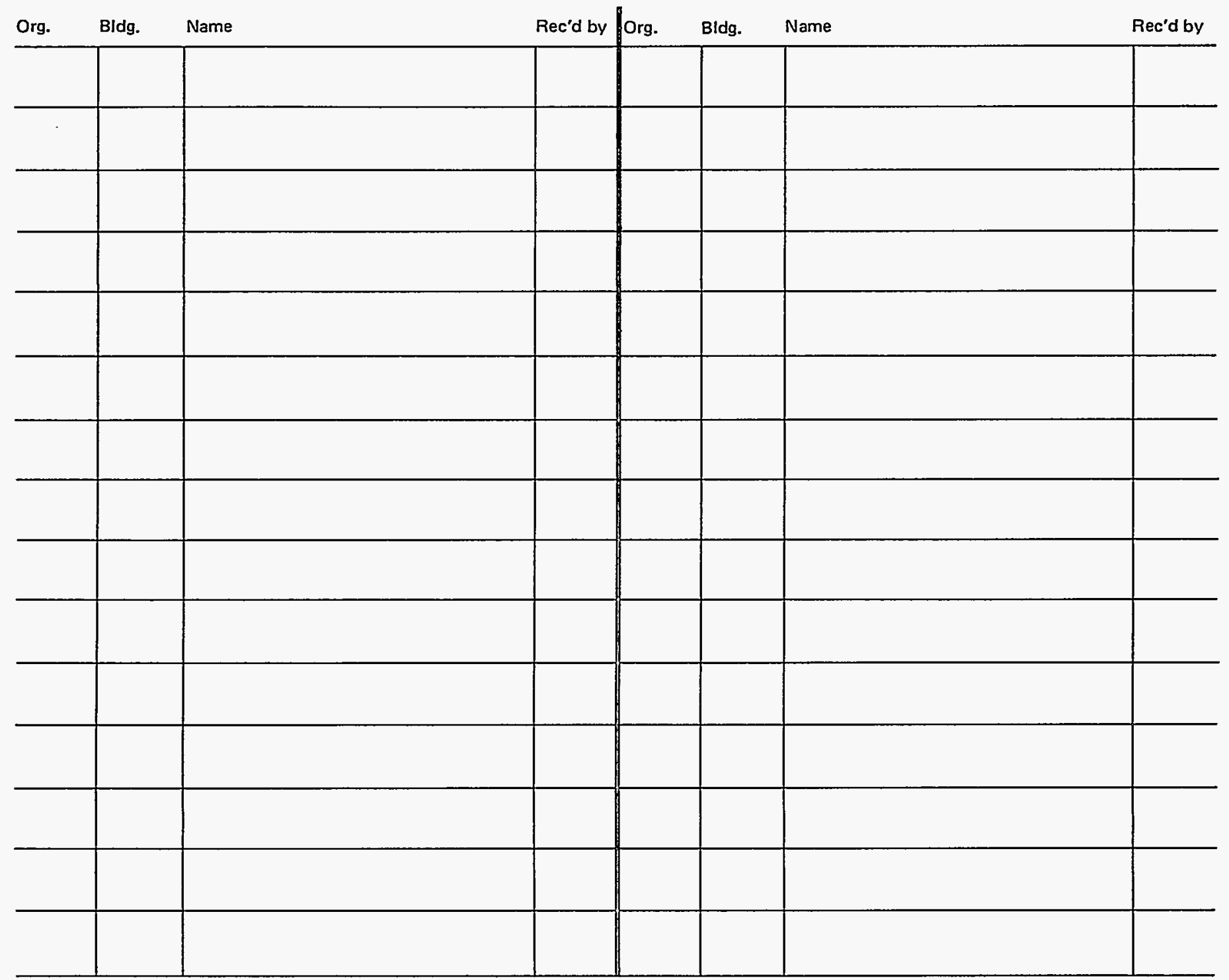

